\title{
REPERTORIO BIBLIOGRÁFICO SOBRE LA COLECCIÓN STUDIEN ZUR ROMANISCHEN SPRACHWISSENSCHAFT UND INTERKULTURELLEN KOMMUNIKATION (1998-2009) DEL GRUPO EDITORIAL PETER LANG \\ Julia Lobato Patricio \\ Universidad Pablo de Olavide de Sevilla \\ Emilio Ortega Arjonilla \\ Universidad de Málaga
}

\section{ABSTRACT}

This bibliographic article collects the books published in the Studien zur romanischen Sprachwissenschaft und interkulturellen Kommunikation Collection by the publishing house Peter Lang. We thus present all the works published so far (1998-2009), representing different domains related to Translation and Interpreting Studies (Translation Theory, Interpreting, Scientific and Technical Translation, Legal Translation, Audiovisual Translation, Literary Translation, Terminology, etc).

KEYWORDS: Bibliography, Translation theory, Interpreting, Medical Translation, Technical Translation, Legal Translation, Audiovisual Translation, Literary Translation, Terminology, Dictionaries.

\section{RESUMEN}

El presente artículo bibliográfico recoge las obras publicadas en la colección Studien zur romanischen Sprachwissenschaft und interkulturellen Kommunikation de la editorial Peter Lang. Así, se presentan todas las obras publicadas hasta la fecha (1998-2009), así como las que están en prensa y en preparación, que representan diversos ámbitos relacionados con los Estudios de Traducción e Interpretación (teoría de la traducción, interpretación, traducción científica y técnica, traducción jurídica, audiovisual, literaria, terminología, etc.).

PALABRAS CLAVE: Bibliografía, Teoría de la traducción, Interpretación, Traducción médica, Traducción técnica, Traducción jurídica, Traducción audiovisual, Traducción literaria, Terminología, Diccionarios. 


\section{INTRODUCCIÓN}

La colección Studien zur romanischen Sprachwissenschaft und interkulturellen Kommunikation de Peter Lang es una de las colecciones de referencia, a escala internacional, dentro de los Estudios de Traducción e Interpretación y disciplinas cercanas (estudios lingüísticos y culturológicos). Comenzó su andadura en 1998, con la publicación de la obra Teoría del campo y semántica léxica de Gerd Wotjak, quien, a su vez, es director de la colección. Gerd Wotjak, es catedrático de Lingüística y Traductología románicas, desde hace más de 30 años, en la Universidad de Leipzig (Alemania). Tiene una amplia experiencia docente durante la cual ha impartido clases en varios países y universidades extranjeras. Ha publicado varios libros y numerosos artículos, entre otros, sobre teoría del léxico y teoría de la valencia, el significado léxico y el potencial comunicativo de lexías, sobre lingüística contrastiva y campos léxicos. La colección cuenta actualmente con más de 70 obras publicadas principalmente en alemán y español, aunque también podemos encontrar trabajos redactados en portugués o en inglés. En la página web www.peterlang.com se puede consultar el resumen de cada una de estas obras.

Entre las características que definen a esta colección internacional de Traducción e Interpretación destaca, entre otras, que es una colección abierta a la publicación en diversos idiomas europeos. Esta apertura a la difusión en varios idiomas es una seña de identidad que la diferencia de otras colecciones internacionales, que son mucho más restrictivas en cuanto a la publicación en según qué lenguas.

Por otro lado, también son dignas de destacar tanto su diversidad temática como la apertura a distintos ámbitos y enfoques traductológicos, tanto teóricos como metodológicos y/o prácticos.

Por último, destacar su vocación universitaria y académica. Es una colección dirigida a Universidades, centros de investigación, alumnos, profesores e investigadores dentro del amplio abanico de ámbitos (o subámbitos) que se engloban dentro de los Estudios de Traducción e Interpretación y disciplinas cercanas (Estudios Lingüísticos, Estudios culturológicos, etc.).

Resulta, a nuestro modo de ver, una colección indispensable para el seguimiento de la producción científica en Traducción e Interpretación, a escala internacional, desde su lanzamiento, en 1998, hasta la fecha. 


\section{REPERTORIO BIBLIOGRÁFICO}

A continuación incluimos un listado de las referencias bibliográficas de todas las obras que podemos encontrar en esta colección desde 1998 hasta 2009.

Número 1

Wotjak, Gerd (ed.), Teoría del campo y semántica léxica Théorie des champs et sémantique lexicale, Frankfurt/M., Berlin, Bern, New York, Paris, Wien, 1998. XII, 354p. ISBN 978-3-631-34026-4.

Número 2

Eckkrammer, Eva Martha / Eder, Hildegund Maria (Cyber)Diskurs zwischen Konvention und Revolution. Frankfurt/M., Berlin, Bern, Bruxelles, New York, Wien, 2000. 344 p. ISBN: 978-3-631-34831-4.

\section{Número 3}

Gräfe, Monika, Untersuchungen zur Konstituentenabfolge spanischer Adverbiale auf Satzund Textebene mit EDV-gestïtrter quantitativer Analyse. Frankfurt/M., Berlin, Bern, Bruxelles, New York, Oxford, Wien, 2000. 364 p. ISBN: 978-3-631-35197-0.

Número 4

Larreta Zulategui, Juan Pablo, Fraseología contrastiva del alemán y el español, Teoría y práctica a partir de un corpus bilingüe de somatismos. Frankfurt/M., Berlin, Bern, Bruxelles, New York, Oxford, Wien, 2001. LVIII, 255 p. ISBN: 978-3-63137343-9.

Número 5

Jiménez Hurtado, Catalina, Léxico y Pragmática. Frankfurt/M., Berlin, Bern, Bruxelles, New York, Oxford, Wien, 2001. 282 p. ISBN: 978-3-631-37942-4.

Número 6

Jiménez Ramírez, Félix, El español en la Suiza alemana. Estudio de las características lingüisticas e identitarias del español de la segunda generación en una situación de contacto de lenguas. Frankfurt/M., Berlin, Bern, Bruxelles, New York, Oxford, Wien, 2001. 165 p. ISBN: 978-3-631-38375-9.

Número 7

Wotjak, Gerd (Hrsg.). Studien zum romanisch-deutschen und innerromanischen Sprachvergleich. Akten der IV. Internationalen Tagung zum romanisch-deutschen und innerromanischen Sprachvergleich (Leipzig, 7.10.-9.10.1999) Frankfurt/M., Berlin, 
Bern, Bruxelles, New York, Oxford, Wien, 2001. XV, 723 p. ISBN: 978-3-63136321-8 br.

Número 8

Störl, Kerstin / Klare, Johannes (Hrsg.). Romanische Sprachen in Amerika. Festschrift für Hans-Dieter Paufler zum 65. Geburtstag. Frankfurt/M., Berlin, Bern, Bruxelles, New York, Oxford, Wien, 2002. 642 p. ISBN: 978-3-63135842-9 br.

Número 9

Calañas Continente, José-Antonio. El dominio léxico Existencia en alemán. Diccionario lexemático-funcional alemán-español del lexicón verbal básico. Frankfurt/M., Berlin, Bern, Bruxelles, New York, Oxford, Wien, 2002. IV, 182 p. ISBN: 9783-631-38755-9.

Número 10

Blaikner-Hohenwart, Gabriele (Hrsg.), Portugiesisch, Papiamentu et al. Salzburger Beiträge zu Prozessen und Produkten der Translation in der Romania. Frankfurt/M., Berlin, Bern, Bruxelles, New York, Oxford, Wien, 2003. 204 p. ISBN: 978-3-631-39397-0.

Número 11

Cuartero Otal, Juan, Cosas que se hacen. Esquemas sintáctico-semánticos agentivos del español. Frankfurt/M., Berlin, Bern, Bruxelles, New York, Oxford, Wien, 2003. 230 p. ISBN: 978-3-631-51590-7.

Número 12

Schreiber, Michael. Vergleichende Studien zur romanischen und deutschen Grammatikographie. Frankfurt am Main, Berlin, Bern, Bruxelles, New York, Oxford, Wien, 2004. 166 p. ISBN: 978-3-631-51432-0 br.

Número 13

Mellado Blanco, Carmen. Fraseologismos somáticos del alemán.Un estudio léxicosemántico. Frankfurt am Main, Berlin, Bern, Bruxelles, New York, Oxford, Wien, 2004. 267 p. ISBN: 978-3-631-51998-1.

Número 14

Vanhoe, Henk. Aspectos de la sintaxis de los verbos psicológicos en español. Un análisis léxico funcional. Frankfurt am Main, Berlin, Bern, Bruxelles, New York, Oxford, Wien, 2004. XII, 344 p. ISBN: 978-3-631-52831-0. 
Número 15

Azpiazu, Susana. Las estrategias de nominalización. Estudio contrastivo del estilo nominal. Frankfurt am Main, Berlin, Bern, Bruxelles, New York, Oxford, Wien, 2004. 343 p. ISBN: 978-3-631-52430-5.

Número 16

Meliss, Meike, Recursos lingüísticos alemanes relativos a «GERÄUSCH» y sus posibles correspondencias en español. Un estudio lexicológico modular-integrativo. Frankfurt am Main, Berlin, Bern, Bruxelles, New York, Oxford, Wien, 2005. 354 p. ISBN: 978-3-631-51215-9.

Número 17

Morera, Marcial. La Complementación Morfológica en Español. Ensayo de Interpretación Semántica. Frankfurt am Main, Berlin, Bern, Bruxelles, New York, Oxford, Wien, 2005. IV, 272 p. ISBN: 978-3-631-53420-5.

Número 18

Bustos Plaza, Alberto, Combinaciones verbonominales y lexicalización. Frankfurt am Main, Berlin, Bern, Bruxelles, New York, Oxford, Wien, 2005. 243 p. ISBN: 978-3-631-52911-9.

Número 19

Peeters, Jean (ed.), On the Relationships between Translation Theory and Translation Practice. Frankfurt am Main, Berlin, Bern, Bruxelles, New York, Oxford, Wien, 2005. 258 p. ISBN: 978-3-631-53442-7 / US-ISBN: 978-0-8204-7663-6 pb.

Número 20

Domínguez Vázquez, María José. Die Präpositivergänzung im Deutschen und im Spanischen Zur Semantik der Präpositionen. Frankfurt am Main, Berlin, Bern, Bruxelles, New York, Oxford, Wien, 2005. 281 p. ISBN: 978-3-631-53312-3 br.

Número 21

Hüsgen, Thomas J. C, Vom getreuen Boten zum nachdichterischen Autor. Übersetzungskritische Analyse von Fernando Pessoas Livro do Desassossego in deutscher Sprache. Frankfurt am Main, Berlin, Bern, Bruxelles, New York, Oxford, Wien, 2005. 192 p. ISBN: 978-3-631-53775-6 br.

Número 22

Wotjak Gerd / Cuartero Otal, Juan (eds.), Entre semántica léxica, teoría del léxico y sintaxis. Frankfurt am Main, Berlin, Bern, Bruxelles, New York, Oxford, Wien, 2005. XIII, 485 p. ISBN: 978-3-631-53207-2. 


\section{Número 23}

Casado Velarde, Manuel / González Ruiz, Ramón / Loureda Lamas, Óscar (eds.), Estudios sobre lo metalingüístico (en español). Frankfurt am Main, Berlin, Bern, Bruxelles, New York, Oxford, Wien, 2005. 292 p. ISBN: 978-3-631-53891-3.

Número 24

Martín de León, Celia, Contenedores, recorridos y metas. Metáforas en la traductología funcionalista. Frankfurt am Main, Berlin, Bern, Bruxelles, New York, Oxford, Wien, 2005. 280 p. ISBN: 978-3-631-54128-9.

Número 25

Oster, Ulrike, Las relaciones semánticas de términos polilexemáticos. Estudio contrastivo alemán-español. Frankfurt am Main, Berlin, Bern, Bruxelles, New York, Oxford, Wien, 2005. 300 p. ISBN: 978-3-631-54291-0.

Número 26

Sánchez Nieto, María Teresa. Las construcciones perifrásticas españolas de significado evaluativo y sus equivalentes alemanes en la traducción. Con ejercicios para la clase de español como lengua extranjera. Frankfurt am Main, Berlin, Bern, Bruxelles, New York, Oxford, Wien, 2005. 223 p., CD-Rom. ISBN: 978-3-631-53794-7.

Número 27

Montaner Montava, María Amparo, Análisis cognitivo-perceptivo de la combinatoria de los verbos de transferencia. Se incluye CD-Rom con actividades para estudiantes de lingüística, lenguas y traducción. Frankfurt am Main, Berlin, Bern, Bruxelles, New York, Oxford, Wien, 2005. 295 p., num. il., con CD. ISBN: 978-3-63153328-4.

Número 28

Curell, Clara, Contribución al estudio de la interferencia lingüistica. Los galicismos del español contemporáneo. Frankfurt am Main, Berlin, Bern, Bruxelles, New York, Oxford, Wien, 2005. 115 p. ISBN: 978-3-631-54512-6.

Número 29

Pamies, Antonio / Rodríguez Simón, Francisca. El lenguaje de los enfermos. Metáfora y fraseología en el habla espontánea de los pacientes. Frankfurt am Main, Berlin, Bern, Bruxelles, New York, Oxford, Wien, 2005. VIII, 165 p. ISBN: 978-3-631-54359-7. 
Número 30

Marcelo Wirnitzer, Gisela. Traducción de las referencias culturales en la literatura infantil y juvenil. Frankfurt am Main, Berlin, Bern, Bruxelles, New York, Oxford, Wien, 2007. VIII, 261 p., num. tablas, 1 CD. ISBN: 978-3-631-54726-7.

Número 31

De Miguel, Elena / Palacios, Azucena / Serradilla, Ana (eds.). Estructuras Léxicas y Estructura del Léxico. Frankfurt am Main, Berlin, Bern, Bruxelles, New York, Oxford, Wien, 2006. 367 p. ISBN: 978-3-631-55002-1.

Número 32

Montoro del Arco, Esteban Tomás. Teoría fraseológica de las locuciones particulares. Las locuciones prepositivas, conjuntivas y marcadoras en español. Frankfurt am Main, Berlin, Bern, Bruxelles, New York, Oxford, Wien, 2006. 302 p. ISBN: 978-3631-55001-4.

Número 33

Matschke, Nicola. Zeitgenössische französische Bibelübersetzungen: exemplarische textlinguistische und stilistische. Übersetzungsprobleme. Frankfurt am Main, Berlin, Bern, Bruxelles, New York, Oxford, Wien, 2006. XIV, 298 p. ISBN: 978-3631-55664-1 br.

Número 34

Aznárez Mauleón, Mónica. La fraseología metalingüistica con verbos de lengua en español actual. Frankfurt am Main, Berlin, Bern, Bruxelles, New York, Oxford, Wien, 2006. 478 p. ISBN: 978-3-631-55820-1.

Número 35

Almela Sánchez, Moisés, From Word to Lexical Units. A Corpus-Driven Account of Collocation and Idiomatic Patterning in English and English-Spanish. Frankfurt am Main, Berlin, Bern, Bruxelles, New York, Oxford, Wien, 2006. 421 p. ISBN: 978-3-631-55759-4 / US-ISBN: 978-0-8204-8740-3 pb.

Número 36

Albelda Marco, Marta. La intensificación como categoría pragmática: revisión y propuesta. Una aplicación al español coloquial. Frankfurt am Main, Berlin, Bern, Bruxelles, New York, Oxford, Wien, 2007. 242 p. ISBN: 978-3-631-56452-3.

Número 37

Vidal Claramonte, María del Carmen África. Traducir entre culturas. Diferencias, poderes, identidades. Frankfurt am Main, Berlin, Bern, Bruxelles, New York, Oxford, Wien, 2007. 125 p. ISBN: 978-3-631-56885-9. 
Número 38

Olímpio de Oliveira Silva, Maria Eugênia. Fraseografía teórica y práctica. Frankfurt am Main, Berlin, Bern, Bruxelles, New York, Oxford, Wien, 2007. 335 p. ISBN 978-3-631-57043-2.

Número 39

Jiménez Hurtado, Catalina (ed.). Traducción y accesibilidad. Subtitulación para sordos y audiodescripción para ciegos: nuevas modalidades de Traducción Audiovisual. Frankfurt am Main, Berlin, Bern, Bruxelles, New York, Oxford, Wien, 2007. 287 p. ISBN: 978-3-631-56761-6.

Número 40

Ortega Arjonilla, Emilio (ed.). El Giro Cultural de la Traducción. Reflexiones teóricas y aplicaciones didácticas. Frankfurt am Main, Berlin, Bern, Bruxelles, New York, Oxford, Wien, 2007. 156 p. ISBN: 978-3-631-57155-2.

Número 41

Álvarez de la Granja, María (ed.). Lenguaje figurado y motivación. Una perspectiva desde la fraseología. Frankfurt am Main, Berlin, Bern, Bruxelles, New York, Oxford, Wien, 2008. 302 p. ISBN: 978-3-631-57419-5.

Número 42

Siebold, Kathrin. Actos de habla y cortesía verbal en español y en alemán. Estudio pragmalingüistico e intercultural. Frankfurt am Main, Berlin, Bern, Bruxelles, New York, Oxford, Wien, 2008. 257 p. ISBN 978-3-631-57191-0.

Número 43

Conde Tarrío, Germán (ed.) Aspectos formales y discursivos de las expresiones fijas. Frankfurt am Main, Berlin, Bern, Bruxelles, New York, Oxford, Wien, 2008. 302 p. ISBN: 978-3-631-57426-3.

Número 44

Mellado Blanco, Carmen (ed.). Colocaciones y fraseología en los diccionarios. Frankfurt am Main, Berlin, Bern, Bruxelles, New York, Oxford, Wien, 2008. 332 p. ISBN: 978-3-631-57421-8.

Número 45

Ruiz Gurillo, Leonor / Padilla García, Xose A. (eds.) Dime cómo ironizas y te diré quién eres

Una aproximación pragmática a la ironía. Frankfurt am Main, Berlin, Bern, Bruxelles, New York, Oxford, Wien, 2009. 483 p. ISBN: 978-3-631-58129-2. 
Número 46

Ainciburu, María Cecilia. Aspectos del aprendiraje del vocabulario Tipo de palabra, método, contexto y grado de competencia en las lenguas afines. Frankfurt am Main, Berlin, Bern, Bruxelles, New York, Oxford, Wien, 2008. 183 p. ISBN: 978-3631-57757-8.

Número 47

Rodríguez Rosique, Susana. Pragmática y Gramática. Condicionales concesivas en español. Frankfurt am Main, Berlin, Bern, Bruxelles, New York, Oxford, Wien, 2008. 437 p. ISBN: 978-3-631-58130-8.

Número 48

Veith, Daniel. Italienisch am Río de la Plata. Ein Beitrag zur Sprachkontakttorscbung. Frankfurt am Main, Berlin, Bern, Bruxelles, New York, Oxford, Wien, 2008. XIV, 229 p. ISBN: 978-3-631-57662-5 br.

Número 49

Corpas Pastor, Gloria. Investigar con corpus en traducción: los retos de un nuevo paradigma. Frankfurt am Main, Berlin, Bern, Bruxelles, New York, Oxford, Wien, 2008. XIV, 297 p. ISBN: 978-3-631-58405-7 en rústica

Número 50

Cardozo, Maurício / Heidermann, Werner / Weininger, Markus J. (eds.). A Escola Tradutológica de Leiprig. Frankfurt am Main, Berlin, Bern, Bruxelles, New York, Oxford, Wien, 2009. XXVIII, 369 p. ISBN: 978-3-631-58199-5 br.

Número 51

Amador Rodríguez, Luis Alexis. La derivación nominal en español: nombres de agente, instrumento, lugar y acción. Frankfurt am Main, Berlin, Bern, Bruxelles, New York, Oxford, Wien, 2009. 425 p. ISBN: 978-3-631-58443-9.

Número 52

Mendívil Giró, José-Luis. Origen, evolución y diversidad de las lenguas. Una aproximación biolingüistica. Frankfurt am Main, Berlin, Bern, Bruxelles, New York, Oxford, Wien, 2009. 239 p. ISBN: 978-3-631-58866-6 enc.

Número 53

Alsina, Victòria / Andújar, Gemma / Tricás, Mercè (eds.), La representación del discurso individual en traducción. Frankfurt am Main, Berlin, Bern, Bruxelles, New York, Oxford, Wien, 2009. VI, 293 p., num. ISBN: 978-3-631-58879-6 enc. 


\section{Número 54}

Bravo Utrera Sonia / Garcia López Rosario (eds.) Estudios de traducción: Perspectivas. Zinaida Lvóvskaya in memoriam. Frankfurt am Main, Berlin, Bern, Bruxelles, New York, Oxford, Wien, 2009. 610 p. ISBN: 978-3-631-58907-6 geb.

\section{Número 55}

Buján López Carlos / Dominguez Vázquez María J. (eds.) Centros y periferias en España y Austria: perspectivas lingüisticas y traductológicas. Frankfurt am Main, Berlin, Bern, Bruxelles, New York, Oxford, Wien, 2009. 180 p. ISBN 978-3-63158955-7 br.

Número 56

Penas M. Azucena/González Rosario (eds) Estudios sobre el texto Nuevos enfoques y propuestas. Frankfurt am Main, Berlin, Bern, Bruxelles, New York, Oxford, Wien, 2009. VI, 489 p. ISBN: 978-3-631-58310-4 br.

Número 57

Monferrer-Sala Juan Pedro / Urbán Ángel (eds.) Sacred Text. Explorations in Lexicography. Frankfurt am Main, Berlin, Bern, Bruxelles, New York, Oxford, Wien, 2009. 328 p. ISBN: 978-3-631-59741-5.

Número 58

Wotjak G / Ivanova V / Tabares Plasencia E / (Hrsg./eds.) Translatione via facienda. Festschrift für Christiane Nord zum 65. Geburtstag. Homenaje a Christiane Nord en su 65 cumpleaños. Frankfurt am Main, Berlin, Bern, Bruxelles, New York, Oxford, Wien, 2009. XII, 394 p. ISBN 978-3-631-59486-5 geb.

Número 59

Alvarado Ortega, $\mathrm{M}^{a}$. Belén. Las fórmulas rutinarias del español: teoría y aplicaciones. Frankfurt am Main, Berlin, Bern, Bruxelles, New York, Oxford, Wien, 2010. 227 p. ISBN 978-3-631-59819-1 enc. ISBN 978-3-653-00248-5 (eBook)

Número 60

Buckingham, Louisa. Las construcciones con verbo soporte en un corpus de especialidad. Frankfurt am Main, Berlin, Bern, Bruxelles, New York, Oxford, Wien, 2009. 207 p. ISBN: 978-3-631-59560-2 geb.

Número 61

Mehlberg, Martin. Weil man es so sagt. Wissenschaftstheoretische und valenzlexikographische Überlegungen zu ausgewäblten Aspekten der lexikalischen 
Variation. Frankfurt am Main, Berlin, Bern, Bruxelles, New York, Oxford, Wien, 2010. XII, 384 p. ISBN: 978-3-631-59754-5 geb.

Número 62

Albi Aparicio, Miguel. La valencia lógico-semántica de los verbos de movimiento y posición en alemán y español. Frankfurt am Main, Berlin, Bern, Bruxelles, New York, Oxford, Wien, 2010. XII, 382 p. ISBN: 978-3-631-58952-6 geb.

Número 63

Martinez Pasamar Concepción (ed.) Estrategias argumentativas en el discurso periodístico. Frankfurt am Main, Berlin, Bern, Bruxelles, New York, Oxford, Wien, 2010. 220 p. ISBN: 978-3-631-59423-0 geb.

Número 64

Gutiérrez Pérez, Regina. Estudio cognitivo-contrastivo de las metáforas del cuerpo Análisis empirico del corazón como dominio fuente en inglés, francés, español, alemán e italiano. Frankfurt am Main, Berlin, Bern, Bruxelles, New York, Oxford, Wien, 2010. 219 p. ISBN 978-3-631-59719-4 enc. ISBN 978-3-653-00240-9 (eBook).

Número 65

García Padrón, Dolores / Fumero Pérez, Maria del Carmen (eds.) Tendencias en linguiistica general y aplicada. Frankfurt am Main, Berlin, Bern, Bruxelles, New York, Oxford, Wien, 2010. 263 p. ISBN: 978-3-631-60411-3 enc.

Número 66

Sánchez Aquilino / Almela Moisés (eds.). A Mosaic of Corpus Linguistics Selected Approaches. Frankfurt am Main, Berlin, Bern, Bruxelles, New York, Oxford, Wien, 2010. VI, 291 p. ISBN: 978-3-631-58789-8 hb.

Número 67

Vidal Claramonte, Maria Carmen África. Traducción y asimetría. Frankfurt am Main, Berlin, Bern, Bruxelles, New York, Oxford, Wien, 2010. 139 p. ISBN: 978-3-631-60520-2 enc.

Número 68

Garcia Bernardo, Ana Maria. Zu aktuellen Grundfragen der Überset₹ungswissenschaft. Frankfurt am Main, Berlin, Bern, Bruxelles, New York, Oxford, Wien, 2010. 131 p. ISBN: 978-3-631-60362-8 geb. 
Número 69

Toledo Báez, Maria Cristina. El resumen automático y la evaluación de traducciones en el contexto de la traducción especializada. Frankfurt am Main, Berlin, Bern, Bruxelles, New York, Oxford, Wien, 2010. 311 p. ISBN: 978-3-631-60360-4 enc.

Número 70

Hernández Socas, Elia. Las Islas Canarias en viajeras de lengua alemana. Frankfurt am Main, Berlin, Bern, Bruxelles, New York, Oxford, Wien, 2010. 338 p. ISBN: 978-3-631-60831-9 enc. 